\title{
Digitale Kooperationen der Qatar National Library
}

Die Teilnahme an kooperativen Informationsinfrastrukturen ist wesentlich für alle modernen Nationalbibliotheken und daher auch für die neue Nationalbibliothek von Katar (QNL) notwendig. Allerdings sind - gerade im Vergleich zu Europa - aktive Bibliothekskooperationen in der arabischen Welt noch unterentwickelt. Der Arab Union Catalog, der in Saudi-Arabien organisiert wird, und der WorldCat von OCLC sind Kooperationsbeispiele aus der Katalogisierung, an der sich einige Bibliotheken der arabischen Welt beteiligen, darunter auch die Nationalbibliothek von Katar. Im nationalen Rahmen erwirbt die QNL seit 2013 in Kooperation mit den Hochschulbibliotheken und Forschungsinstitutionen von Katar Nationallizenzen für internationale und regionale Datenbanken. Bis 2017 sind insgesamt mehr als 150 Datenbanken lizensiert, darunter Springer, Web of Science und mehrere arabischsprachige. Die wichtigsten Kooperationsprojekte für Katars Nationalbibliothek sind zwei Digitalisierungsprojekte, die sie mit der Library of Congress und der British Library durchführt.

\section{Kurze Darstellung der neuen Nationalbibliothek von Katar}

Die neue Nationalbibliothek von Katar ist ein Projekt der Qatar Foundation für Bildung, Wissenschaft und gesellschaftliche Entwicklung, einer Bildungsstiftung, die neben modernen Schulen und internationalen Hochschulen auch Krankenhäuser, Kongresscenter, eine hoch spezialisierte Zucht von Araberpferden und weitere Modernisierungen im Staat Katar initiiert und fördert. Für die Bildungsstadt im Osten von Doha, die zahlreiche amerikanische Universitäten angeworben hat, sollte eine neue zentrale Universitätsbibliothek errichtet werden. Das Gebäude wurde 2009 vom Stararchitekten Rem Koolhaas entworfen, der eine Fläche von 45000 Quadratmetern und Raum für 1,2 Millionen Medien vorsah und damit eine neue Bibliotheksdimension im Land begründete. Im November 2012 wurde das Projekt, von dem nur der Bauzaun zu sehen war, in einer öffentlichen Veranstaltung vor Bibliothekaren aus der Golfregion zur Nationalbibliothek erklärt. Die Nationalbibliothek sollte zugleich die Funktio- 
nen einer zentralen Universitätsbibliothek und einer öffentlichen Bibliothek übernehmen. Vom Personal bis zum elektronischen und realen Bestand musste alles von Grund auf neu beschafft und entsprechende Dienstleistungen mussten entwickelt werden. Außerdem wurden Struktur und Abläufe der einzelnen Geschäftsgänge sowie Standards und Normen für die neue Bibliothek festgelegt. Weiterhin musste die Bauplanung in verschiedenen Bereichen der veränderten Aufgabe angepasst werden. Zwischen 2013 und 2017 wurde der Grundbestand an gedruckten Werken in Englisch und Arabisch beschafft, sodass mit der Testöffnung des Gebäudes im November 2017400000 Bände den offenen Freihandbereich füllen und den ersten Nutzern zur Verfügung stehen, hinzukommen weitere 200000 Bände im Magazin im Untergeschoss.

Die neue Nationalbibliothek führt einen großen historischen Bestand. Es ist die frühere Privatbibliothek eines Sheikhs, der seit Ende der 1970er Jahre Bücher und Handschriften gesammelt hat. In Ermangelung ausreichender schriftlicher historischer Zeugnisse in Buchform aus und über Katar erstreckte sich sein Sammeleifer dabei über die gesamte Region. Diese Sammlung von mehr als 73000 historischen Bänden ist ein besonderer Schatz der Nationalbibliothek von Katar. Neben Reiseberichten über die arabische Welt umfasst sie frühe arabische Handschriften zu religiösen und weltlichen Themen, speziell den Naturwissenschaften, frühe Drucke arabischer Wissenschaftler in europäischen Sprachen, vor allem in Latein, historische Karten der arabischen Welt, erste Drucke in arabischer Schrift sowie historische Bücher über die gesamte Region. Darunter befinden sich einige Raritäten, die bei der Eröffnung im April 2018 im Bereich der historischen Sammlung ausgestellt werden. Diese für die Golfregion außergewöhnliche Sammlung enthält darüber hinaus Werke und eigene Bücher eines früheren Emirs von Katar aus den 1960er Jahren.

\section{Gründe für die digitalen Kooperationen der Nationalbibliothek Katars}

Der Schwerpunkt der internationalen digitalen Kooperation der Nationalbibliothek von Katar liegt auf der Digitalisierung und Erschließung von Quellen über den arabischen Raum, insbesondere die Golfregion, und deren internationaler Verbreitung. Um die besten Stücke der eigenen historischen Sammlung digital verfügbar $\mathrm{zu}$ machen und zugleich die arabische Golfregion mit ihren Kulturschätzen weltweit bekannter zu machen, ging die Nationalbibliothek von Katar eine Kooperation mit der World Digital Library (WDL) ein. Dies war vor allem zu 
einem frühen Zeitpunkt wichtig, da die QNL selbst bis 2014 nur beschränkte Digitalisierungsmöglichkeiten besaß und die digitalisierten Werke zunächst noch nicht in einem Portal zur Verfügung stellen konnte. Hier bot sich neben der Kooperation mit der Library of Congress in Washington eine digitale Kooperation mit der British Library an, die mit dem India-Office-Archiv Millionen von Dokumenten über die Golfregion besitzt. Parallel dazu prüft die Nationalbibliothek von Katar die Möglichkeit, mit anderen Institutionen, z. B. dem Vatikan, dem Königlichen Archiv in den Niederlanden, einigen spanischen Bibliotheken mit arabischem Bestand, der Staatsbibliothek zu Berlin sowie einem Archiv in Portugal, ähnliche digitale Kooperationen zu entwickeln. Welche dieser Kooperationen gelingen, ist derzeit - bis auf den Vatikan, mit dem eine wertvolle arabische Karte digitalisiert werden wird - nicht abzusehen. Absicht ist es, bisher noch nicht digitalisierte Archivalien über die Region und wertvolle orientalische Manuskripte und Bücher bereitzustellen. Dahinter steht die Hoffnung der QNL bzw. der Qatar Foundation, dass dann mehr Material für eine bessere wissenschaftliche Erforschung der Geschichte der arabischen Welt sowie des Islam frei im Netz verfügbar ist und darüber die weitere unabhängige Forschung angeregt wird. Daher werden nur Projekte gestartet, die auf der Grundlage von Open Access den Zugang zu den digitalisierten Werken ermöglichen.

\section{Die Kooperation mit der World Digital Library}

Die WDL verfolgt das Ziel, wichtige Werke der Weltkultur aus jeder Nationalbibliothek oder anderen Bibliotheken exemplarisch für die jeweilige Kultur digital verfügbar zu machen und in allen UNESCO-Sprachen ihre Bedeutung für die Kultur des jeweiligen Landes oder der jeweiligen kulturellen Gruppe zu erläutern. Weiterhin sollen in der WDL alle UNESCO-Welterbe-Objekte online zugänglich werden. Das Projekt ging 2005 von der Library of Congress aus und fand sehr schnell weltweit weitere Partner. 2009 wurde zwischen der Qatar Foundation und der Library of Congress eine Vereinbarung zur Entwicklung der WDL getroffen, die im Mai 2010 durch ein Memorandum of Understanding noch betont wurde. Die Webseite der WDL wurde im April 2009 im UNESCO-Hauptquartier in Paris vorgestellt und Sheikha Mozah Bint Nasser Al-Missned, die Vorsitzende der Qatar Foundation, sendete eine Grußbotschaft per Videokonferenz und verdeutlichte damit die Unterstützung aus Katar.

Die Nationalbibliothek von Katar, damals noch Zentralbibliothek, lieferte zur Gründung der WDL erste Digitalisate von arabischen Materialien mit Metadaten und Erläuterungen. Als Institution der Qatar Foundation förderte sie die 
Library of Congress beim Aufbau der WDL von 2010 bis 2016 finanziell und war einer der wichtigsten Sponsoren. ${ }^{1}$ Dabei ging es zunächst darum, das Gesamtkonzept einer WDL für UNESCO zu unterstützen und über die Beteiligung der QNL im Exekutivkommitee der WDL das Projekt in der Golfregion zu verstetigen.

Die Vereinbarung zwischen der Qatar Foundation, der Nationalbibliothek und der Library of Congress zielte vor allem auf diese finanzielle Unterstützung, um das Projekt technisch und organisatorisch aufzubauen und die Kooperation anderer Golfstaaten mit der WDL anzuregen. Da die WDL die Webseiten in den sieben UNESCO-Sprachen sowie in Portugiesisch veröffentlicht, ist Arabisch ein Teil des Projekts. Damit kann jeder Teilnehmer an der WDL interessante digitale Objekte und deren Beschreibungen auch für die gesamte arabische Welt verfügbar machen. Ein anderer Punkt der Vereinbarung bestand darin, im gemeinsamen Projekt arabische und islamische Manuskripte der Library of Congress zu digitalisieren und mit den entsprechenden Metadaten und wissenschaftlichen Kommentaren $\mathrm{zu}$ versehen. Weiterhin vereinbart wurde die aktive Rolle der Nationalbibliothek von Katar im Sinne einer Vorbildfunktion für andere Golfstaaten. 354 Objekte der QNL, vor allem historische Karten und Manuskripte, wurden in die WDL aufgenommen. ${ }^{2}$ Dazu stellte die Nationalbibliothek beispielhaft erste Digitalisate wertvoller Handschriften und Karten zur Verfügung, z. B. die Sexta Asiae tabula, eine Karte nach Ptolemäus aus dem 2. Jahrhundert, gedruckt 1478, auf der die arabische Halbinsel mit einem Ort Katara abgebildet ist, von dem angenommen wird, dass er Katar bezeichnet. Das Original gehört zu den wertvollsten Werken der historischen Sammlung der QNL. ${ }^{3}$ Mit diesem Beitrag steht die Nationalbibliothek von Katar an zweiter Stelle der Zulieferer für die WDL aus der MENA-Region und an 13. Stelle aller 183 weltweiten Partner.

Die digitale Kooperation mit der WDL diente der Qatar National Library da$\mathrm{zu}$, einen internationalen Standard für Digitalisierung und die Erstellung von Metadaten in der eigenen Region einzuführen und die Ergebnisse in einem Webportal zugänglich zu machen. Zu Beginn verfügte die QNL noch nicht über ein solches Portal, sie erhielt dies erst als Ergebnis der ersten Phase der digitalen Kooperation mit der British Library. Bei zunächst nur wenig Personal in der Nationalbibliothek von Katar war die professionelle Unterstützung der Library

1 Vgl. dazu Oudenaren, John Van: The World Digital Library. In: Bibliothek - Forschung und Praxis 41 (2017) H. 2. S. 242-249.

2 Vgl. https://www.wdl.org/en/search/?q=Qatar+National+Library (22.12.2017).

3 Sixth Map of Asia: https://www.wdl.org/en/item/2702/\#regions=middle-east-and-north-africa\&countries=QA (22.12.2017). 
of Congress mit ihren Fachleuten äußerst wichtig. Diese kamen regelmäßig zu der von der QNL organisierten Fortbildung zur WDL für die Golfregion. Hier lud die Bibliothek ihre Partner aus den umliegenden Staaten Jemen, Oman, UAE, Bahrain, Saudi-Arabien, Kuwait, Sudan und Somalia ein, um sie über aktuelle Digitalisierungstechniken und $\mathrm{zu}$ damit verbundenen Themen $\mathrm{zu}$ informieren und sie zur Teilnahme an der WDL zu motivieren. Diese Seminare fanden als Treffen der Arab Peninsula Group von 2010 bis 2016 jährlich im Frühjahr statt. Vertreter der Library of Congress und weitere internationale Spezialisten referierten zu verschiedenen Aspekten der Digitalisierung, der digitalen Objekte und der WDL. Die Vorträge wurden in Englisch oder Arabisch gehalten und simultan übersetzt. Nachdem die QNL ihr erstes Digitalisierungslabor eingerichtet hatte, kamen auch praktische Übungen hinzu. So konnte sie den Bibliotheken und Informationseinrichtungen, die über keine hoch entwickelte technische Ausstattung verfügten, Anregungen und Hilfestellung geben. Großen Wert legte man auf die Vermittlung der internationalen Standards für die Digitalisierung, die Technologie und Anbieter sowie auf die Erstellung der Metadaten, worum sich das zweite Seminar im Jahr 2012 drehte. 2013 ging es um Open Access und Copyright, wobei zugleich lebhaft über Open Access in der Region diskutiert wurde. 2014 handelte das Seminar vom kulturellen Erbe und seiner Bewahrung. Im Frühjahr 2015 gab es eine praktische Unterweisung im ausgebauten Digitalisierungszentrum der QNL sowie die Präsentation der Qatar Digital Library, des eigenen frei verfügbaren Portals der QNL, das inzwischen aus der Kooperation mit der British Library hervorgegangen war. Im Jahr 2016 wurde schließlich die finanzielle Unterstützung der WDL eingestellt, jedoch die aktive Teilnahme der Nationalbibliothek von Katar an der WDL fortgesetzt.

\section{Die Kooperation mit der British Library}

Hintergrund für die digitale Kooperation mit der British Library ist, dass die QNL ihre digitalen Strukturen parallel zu ihrem analogen Grundbestand aufbauen musste, allerdings nur sehr wenige Archivalien über Katar und die Region besaß. Als Nationalbibliothek will sie jedoch eine angemessene und bedeutende Sammlung zu Katar anbieten. Daher erschien eine digitale Kooperation mit denjenigen, die historische Dokumente über die Region besitzen, sie gerne digitalisieren und den Originalbestand bewahren wollten, eine gute Lösung für beide Seiten - für die Nationalbibliothek von Katar, der solche Dokumente fehlten, und die Partner, die ihre Dokumente digitalisieren möchten. 
Die Golfregion wurde lange Zeit (1822-1972) von den Engländern kontrolliert, zuvor beherrschten der Oman, Holland und Portugal wichtige Stützpunkte in der Region. Auch die wirtschaftlichen Beziehungen mit Indien oder Persien trugen entscheidend zur Geschichte der Golfregion bei. Und da schließlich die Nationalbibliothek von Katar selbst kaum über Originaldokumente verfügt, sind die Archivalien aus diesen Ländern von großem Interesse. Die wichtigste Ressource für die letzten zwei Jahrhunderte britischer Herrschaft im Arabischen Golf sind die India Office Records (IOR), ein Archiv, das heute in der British Library in London lagert. Es enthält die Archivalien der OstindienKompanie (1600-1858), der Ausschüsse der Beauftragten für Angelegenheiten zu Indien (1784-1858) und des Indien-Büros (1858-1947). Zusätzlich umfasst es kleinere private Briefe und Sammlungen über die britische Herrschaft im indischen Raum. ${ }^{4}$ Obwohl das Gesamtarchiv mit einer Länge von 14 Kilometern bis dahin nur grob erschlossen war, gab es einige Hinweise auf wichtige historische Quellen für Katar und die umliegende Golfregion. Erst durch die digitale Kooperation zwischen der Nationalbibliothek von Katar und der British Library konnte eine systematische Erschließung, Digitalisierung und schließlich Präsentation in der Qatar Digital Library realisiert werden. Gleichzeitig wird die British Library damit ihren Bestand im eigenen Webportal präsentieren können.

Am Rande eines Staatsbesuches des Emirs von Katar, Sheikh Hamad Bin Khalifa Al Thani, in London im Jahr 2010 unterzeichnete seine Frau Sheikha Mozah Bint Nasser Al-Missned ein Memorandum der von ihr geleiteten Qatar Foundation mit der British Library. Ziel dieser über zehn Jahre angelegten Vereinbarung ist die Digitalisierung wichtiger Archivmaterialien über die Golfregion und arabischer Manuskripte der British Library für die Qatar National Library. Nach intensiven Verhandlungen von Mai bis Mitte Juli 2012 konnte der Vertrag über die erste Phase des Projekts am 18. Juli 2012 in London unterzeichnet werden. In dieser ersten Phase der Partnerschaft richtete die British Library ein Digitalisierungszentrum in einer Etage ihres Gebäudes in St. Pancras ein und stellte 43 qualifizierte Personen für das Projekt ein. Angestrebt war, von Juli 2012 bis Ende 2014475000 relevante Seiten aus dem India-Office-Archiv und 25000 Seiten orientalischer Karten und Manuskripte der British Library zu digitalisieren. Ebenso wurden einige Audio- und Videodateien sowie mehrere wissenschaftliche Artikel, die die digitalisierten Werke erläutern und in einen historischen Rahmen stellen, produziert. So können die Inhalte auch einem nicht wissenschaftlichen Publikum nahegebracht werden. Weiterhin sollte ein Web-

4 Vgl. Marzo, Flavio, Anna Hoffman u. Barbara Borghese: The British Library/Qatar Foundation behind the scenes - an interview with $\mathrm{NiC}$, 25. Oktober 2013. https://www.iiconservation. org/node/4390 (27.12.2017). 
portal aufgebaut werden, das diese Digitalisate frei verfügbar macht und der Nationalbibliothek von Katar zusätzlich die Möglichkeiten bietet, ihre eigenen Digitalisate dort einzubringen. Die Dokumente bleiben in der Originalsprache, werden aber mit zweisprachigen Metadaten versehen; ebenso werden die inhaltlichen Erläuterungen in Englisch und Arabisch erstellt, sodass im Portal in beiden Sprachen gesucht werden kann. Im Portal soll alles als Open Access veröffentlicht werden - immerhin lautet das Ziel der Qatar Foundation, freien $\mathrm{Zu}$ gang zu mehr Informationen über die arabische und islamische Welt bereitzustellen und derart mehr Forschung darüber anzuregen. Was die Manuskripte betrifft, so werden vorrangig naturwissenschaftliche Werke der arabischen Blütezeit im Mittelalter digitalisiert, da diese besonders gut den Einfluss der arabischen Wissenschaft auf die Renaissance in Europa bezeugen.

In dieser ersten Phase kam es darauf an, bestimmte Bereiche des IndiaOffice-Archivs auf relevante Dokumente durchzusehen und diese für die Nationalbibliothek von Katar zu digitalisieren. Die Engländer hatten in der Golfregion zwei zentrale Stützpunkte: Bushire, das im heutigen Iran liegt, und Bahrain. Der Schwerpunkt der zu digitalisierenden Archivalien lag daher auf den Dokumenten zur Arabischen Halbinsel und zum Arabischen Golf, die etwa zwischen 1745 und 1947 von der früheren East India Company oder den genannten Residenzen des britischen Reiches erstellt wurden, darunter auch Briefe und Tagebücher. Lorimers Gazetteer, ein berühmtes mehrbändiges Werk, das Geheimberichte an die britische Regierung über den Persischen Golf, Oman und Zentral-Arabien in der Zeit bis 1915 enthält, wurde ebenfalls in der ersten Phase des Projekts digital verfügbar gemacht.

In dieser Kooperation ging man zunächst von schon bekannten Bereichen des Archivs aus, die aber noch nicht im Detail gesichtet worden waren. So konnten wichtige Dokumente über frühere Emire von Katar sowie zur Ölförderung im Land gefunden werden. In mehreren gemeinsamen Sitzungen besprachen die Projektpartner nicht nur die Inhalte, vielmehr wurde das Portal auch den Anforderungen und Wünschen der Nationalbibliothek von Katar angepasst. Am 22. Oktober 2014 wurde dieses Portal - als Ergebnis der digitalen Kooperation der British Library mit der QNL - mit einem Festakt in der Bildungsstadt in Katar eröffnet. ${ }^{5}$ Innerhalb des ersten Monats verzeichnete das Portal mehr als 780000 Pageviews $^{6}$ vor allem aus der Region (Saudi-Arabien, Ägypten, Oman,

5 Vgl. New Qatar Digital Library Offers Readers Unrivalled Collection of Precious Heritage Material. Pressemitteilung der Qatar Foundation vom 22. Oktober 2014. https://www.qf.org. qa/news/digital-library-offers-readers-unrivalled-collection-of-precious-heritage (27.12.2017). 6 Vgl. Qatar Foundation - Qatar National Library: Second Phase of Qatar Foundation, Qatar National Library and British Library launched, London, UK, 19. Januar 2015. https://www.qf. org.qa/app/media/28245 (3.1.2018). 
Kuwait, Bahrain, UAE und Katar), den USA und Europa, wo Hochschulen dieses neue Angebot intensiv nutzten. Bis Juli 2017 blieb die Nutzung mit 9000 bis 13000 Pageviews täglich stabil, sank allerdings im Zusammenhang mit der Blockade Katars durch die UAE, Saudi-Arabien, Ägypten und Bahrain, die auch den Zugang zu den katarischen Webseiten sperrten, im August auf unter 3000. ${ }^{7}$

Für das gemeinsame Projekt wurde gerade am Anfang besonderer Wert auf eine aktive Pressearbeit gelegt, die sogar Bestandteil des Vertrags der ersten Phase war. Erwähnenswert sind die Tales from the India Office von Matthew Teller, der verschiedene Digitalisate heraushebt. ${ }^{8}$ Auch auf YouTube und bei Twitter unter @BLQatar werden bis heute regelmäßig Tweets zu neu entdeckten und digitalisierten Materialien veröffentlicht.

Ein anderer Aspekt der digitalen Kooperation zwischen diesen zwei Nationalbibliotheken umfasst die notwendige Klärung von Copyrightfragen der späteren Privatdokumente sowie die Einbettung der Bestandserhaltung in den Geschäftsgang. ${ }^{9}$ In der British Library findet nicht nur die Analyse der Archivalien und die Durchsicht auf relevantes Material für den Zweck des Projekts statt - außerdem die Erstellung der Metadaten, die Digitalisierung mithilfe unterschiedlicher Geräte bis hin zur Spezialfotografie -, sondern es gibt auch einen Spezialraum für die Bestandserhaltung, der für die Behandlung der Papiere aus den Sammlungen ausgerüstet ist. Hier wird jedes Stück daraufhin untersucht oder vorbereitet, ob und wie es digitalisiert werden kann, ohne dass das Werk Schaden erleidet. Es wird bestimmt, in welcher Weise das Papier vorher oder anschließend behandelt werden muss, damit das Werk langfristig in der Sammlung aufbewahrt und gegebenenfalls auch als Original benutzt werden kann. ${ }^{10} \mathrm{Zu}$ erwähnen ist weiterhin die computergestützte Geschäftsroutine für diese Art der komplexen Massendigitalisierung. Die Abbildung jedes Schrittes und der Überblick in einem System, wie weit man sich den geplanten Ergebnissen bereits angenähert hatte, ermöglicht es der British Library, die von der Nationalbibliothek von Katar aufgestellten Digitalisierungszahlen tatsächlich zu erreichen. Es gab dabei nur geringe Probleme, die schnell behoben wurden. Das Kontrollsystem kann die QNL für ihre eigenen Digitalisierungsprojekte implementieren und nutzen.

Nach guten Ergebnisse und steigender Nutzung der Qatar Digital Library in den ersten Wochen begann 2015 die zweite Phase der zehnjährigen Koope-

7 Vgl. https://www.easycounter.com/report/qdl.qa (27.12.2017).

8 Teller, Matthew: Tales from the India Office. BBC News vom 22. Oktober 2014. http://www. bbc.com/news/magazine-29702694 (28.12.2017).

9 Vgl. Marzo/Hoffman/Borghese, The British Library/Qatar Foundation (wie Anm. 4). 10 Vgl. Marzo/Hoffman/Borghese, The British Library/Qatar Foundation (wie Anm. 4). 
ration. ${ }^{11}$ In dieser Phase des Digitalisierungsprojektes der Nationalbibliothek von Katar und der British Library werden in vier Jahren, von Januar 2015 bis Dezember 2018, insgesamt 1125000 Seiten digitalisiert und in der Qatar Digital Library verfügbar gemacht, darunter 970000 Seiten aus dem India-OfficeArchiv zwischen 1850 und 1951, auch historische Karten und Fotos, da wir bei den Nutzern des Portals großes Interesse an Fotografien und Karten festgestellt haben. Zusätzlich werden 100000 Seiten privater Aufzeichnungen digitalisiert, etwa jene von Anne Blunt, die wohl als erste Frau die Arabische Wüste durchquert hat. 56000 Seiten werden von weiteren, vorwiegend naturwissenschaftlichen arabischen Handschriften aus dem Besitz der British Library eingebracht. Durch die gute personelle Besetzung der historischen Sammlung und die nunmehr bessere Kenntnis der Archive konnten diesmal in mehreren Besprechungen die konkreten Bedarfe der Nationalbibliothek von Katar stärker als zuvor miteingebracht und der $\mathrm{zu}$ digitalisierende Inhalt besser definiert werden. Ebenso werden in der zweiten Phase einige notwendige Verbesserungen im Portal umgesetzt. Auch die zweite Phase verläuft gegenwärtig erfolgreich. Aktuell (7. Januar 2018) enthält die Qatar Digital Library 1240829 Seiten und ist damit für die arabische Welt, speziell für die Golfregion, schon jetzt ein außergewöhnliches und wertvolles historisches Portal.

\section{Digitale Kooperationen als Win-win-Situation für die Partner}

Die Digitalisierungskooperationen der Nationalbibliothek von Katar unterscheiden sich deutlich von Kooperationen für die Europeana oder andere digitale Bibliotheken. Auf der einen Seite konnte sich in einer Win-win-Situation die WDL durch die besondere Förderung der Qatar Foundation technisch und inhaltlich gut entwickeln. Auf der anderen Seite befindet sich hier eine Nationalbibliothek im Aufbau, die kaum über eigene Werke oder Digitalisate verfügt, aber mit Geld ein internationales Projekt wie die WDL unterstützen und sich damit selbst in die digitale Welt katapultieren kann. Durch diesen Einsatz wurde die neue QNL nicht nur international sichtbar, sondern erreichte auch ihre Ziele, mehr arabische Inhalte im Web darzustellen und Menschen auf die arabische Kultur sowie

11 Vgl. https://www.qf.org.qa/app/media/28245 (wie Anm. 6) und Second Phase of QF, Qatar National Library and British Library Partnership Launched. Pressemitteilung der Qatar Foundation vom 19. Januar 2015. https://www.qf.org.qa/news/second-phase-of-qf-qnl-and-british-library-partnership-launched (3.1.2018). 
ihren wissenschaftlichen Beitrag in den Bereichen Medizin, Physik, Mathematik und in anderen Natur- und Geisteswissenschaften im Mittelalter aufmerksam zu machen.

Die Nationalbibliothek von Katar profitiert vom Projekt mit der British Library, die einen ungehobenen Schatz der Geschichte der Golfregion bewahrt. Der Vorteil für die British Library ist offensichtlich, kann sie doch damit das Archiv erschließen und zugleich noch andere Partner für ähnliche Kooperationen gewinnen, da sie ihre Kompetenz in diesem Projekt der Massendigitalisierung bewiesen hat. Durch die Digitalisierung werden für Katar neue historische Dokumente erschlossen. Dies geschieht außerdem auch für die umliegenden Länder, wodurch die Nationalbibliothek von Katar schon vor der Eröffnung des imposanten neuen Gebäudes im April 2018 eine besondere Bedeutung in der Region erhielt. Mit der Qatar Digital Library hat die Nationalbibliothek von Katar zudem eine neue und komfortable Recherchemöglichkeit für die Forschung über die Golfregion, die Arabische Halbinsel und arabische Manuskripte geschaffen, die mit Sicherheit langfristig viele neue Ergebnisse produziert. 